\title{
USER EXPERIENCE STUDY ON IDEATING WEARABLES IN VR
}

\author{
Van Goethem, Sander; \\ Verlinden, Jouke; \\ Watts, Regan; \\ Verwulgen, Stijn \\ University of Antwerp, Ambtmanstraat 1, 2000 Antwerpen
}

\begin{abstract}
More than ever, the ability to quickly and effectively shape ideas in a 3D environment is essential for industrial designers and with the rise of XR technology, a shift from traditional, screen-based CAD to VR-based CAD could improve time to market and personal effectiveness for product designers. In this study, this shift is assessed from a user experience perspective. Ten professional product developers are asked to design respirator masks. The experiment takes place in the Gravity Sketch VR app, using a HTC Vive Pro HMD. The participants are observed, surveyed and interviewed regarding different parameters on their experience. Participants experienced VR Aided Design as quick and intuitive. They personally felt performant and they enjoyed the process. As of now, VRAD is not seen as an alternative to neither sketching nor CAD. Instead, new users experience it being a new tool that can be positioned either parallel to or in between ideation sketching, clay modeling and detailed CAD design. Lastly, this paper includes a preliminary look at a VR Stylus for virtual reality aided design.
\end{abstract}

Keywords: Virtual reality, Design practice, VRAD, Computer Aided Design (CAD)

\section{Contact:}

Van Goethem, Sander

University of Antwerp

Product Development

Belgium

Sander.VanGoethem@uantwerpen.be

Cite this article: Van Goethem, S., Verlinden, J., Watts, R., Verwulgen, S. (2021) 'User Experience Study on Ideating Wearables in VR', in Proceedings of the International Conference on Engineering Design (ICED21), Gothenburg, Sweden, 16-20 August 2021. DOI:10.1017/pds.2021.595 


\section{INTRODUCTION}

For industrial designers, going from pencil sketching straight to screen based Computer Aided Design CAD) has been the standard for two decades (Tovey, 1997). Lately, design researchers have been looking into Virtual Reality (VR) and its advantages for industrial design (Berg and Vance, 2017). The usefulness of VR for early stage concept design has already been touched on by many (Adenauer et al., 2013). It stimulates spatial ability during 3D sketching (Israel et al., 2009), creativity in technology education (Thorsteinsson and Page, 2007) and efficiency in assembly (Nee et al., 2012). After all it makes sense to design a 3D concept directly in a $3 \mathrm{D}$ environment instead of on a $2 \mathrm{D}$ interface, e.g. a computer monitor.

Currently, available solutions for VR-based CAD design are 3D sketching applications that employ standard VR controllers. Although ergonomically shaped, these controllers are meant to be used for gaming purposes. Researchers have proposed other controllers and/or user interfaces (UIs) specifically designed for the purpose of drawing and 3D modeling in Mixed Reality (MR). Two types of alternative controllers are firstly the use of hand-gestures: with haptic devices (Bakker et al., 2017; Bordegoni et al., 2010), haptic gloves (Schkolne et al., 2001) or bare handed (Chu et al., 1997; Hummels and Stappers, 1998; Krichenbauer et al., 2018; Piumsomboon et al., 2013) and secondly the use of stylus-shaped controllers: on physical planes (Fiorentino et al., 2002; Reipschläger and Dachselt, 2019), on tablets (Bae et al., 2008; Drey et al., 2020; Wang et al., 2019), on physical prototypes (Milosevic et al., 2016) or in the air (Jackson and Keefe, 2016; Sachs et al., 1991). Until recently, these alternatives have not found their way into the commercial market. Logitech S.A. recently changed this this by launching their VR Ink stylus (Pilot Edition) (Logitech, n.d.).

The potential for this 3D design environment becomes most visible when shaping wearable products that have organic surfaces (Van Goethem et al., 2020; Nunes et al., 2017), e.g. backpacks, shin guards or sneakers. However, few researchers have looked at immersive 3D modeling from the designers' point of view. In other words, looking at the act of 3D sketching and its intrinsic effect on parameters like efficiency, creativity, effectiveness, and personal enjoyment in 3D design. In product development, the ideation phase usually consists of generating a large variety concepts, traditionally using pencil sketching, clay or foam sculpting. Immersive alternatives for ideation are VR sketching applications like Google Tilt Brush (Cass, 2019) or Oculus Quill (Quill, n.d.). However, in our experience, the current gold standard for the designerly use of VR, is Gravity Sketch VR (Couche et al., 2014). For example, Gravity Sketch is already being implemented in the automotive industry (Lawson et al., 2016).

The most recent work from a designers perspective was by Joundi et al. (Joundi et al., 2020), who used master students to gain insights on Gravity Sketch VR. Their conclusion is twofold: Firstly, VR can be a helpful tool for visualizing and 3D modeling enclosed shapes and secondly, Gravity Sketch VR still has a steep learning curve. D.S. Özgen (Özgen et al., 2019) uses Google Blocks to prove that VR can boost personal effectiveness and personal enjoyment in architecture students. Rieuf et al (Rieuf et al., 2017) and Häggman et al (Häggman et al., 2015) compared the traditional design process to the immersive process aesthetically and semantically. Balzerkiewitz and Stechert (Balzerkiewitz and Stechert, 2020) provided a good overview of current consumer-level VR sketching apps, illustrating that for researching VR Aided Design (VRAD) there is no longer a need for design researchers to develop their own applications (Bourdot et al., 2010; Fuge et al., 2012; Ingrassia and Cappello, 2009). Maurya et al provide a novel approach to concept generation, prototyping and storyboarding with Mixed Reality (Maurya et al., 2019). A. Oti and N. Crilly looked at overcoming the challenges of paper-based designing using VRAD (Oti and Crilly, 2020) from a spatial recognition perspective.

In summary, researchers agree that VRAD can be a helpful tool for designers. There is evidence that VRAD can boost personal effectiveness, enjoyment, and spatial recognition. Secondly, there is some conflict about whether or not VRAD has a steep learning curve (Van Goethem et al., 2020; Joundi et al., 2020). Lastly, researchers have been looking into ways of optimizing the VRAD workflow by prototyping different controllers. By researching designers' experiences with VRAD, this paper aims to provide additional evidence about the abovementioned parameters. The overarching research question for this paper is: 'How do product designers experience trying out VR-based ideation for the first time?'. The participants are chosen to have master's degree in industrial design, while still being young and flexible towards technological advancements. This experience will be referenced against their personal workflow. Knowing that trained product developers know already what their usual workflow looks like, quantitative comparison to traditional media was deemed unnecessary. The experiment was carried out 
with VR novices for two reasons: Firstly, VRAD is still too new to define and find VR 'experts' and secondly, this would allow testing whether VRAD would be deemed 'easy to learn' by participants. Lastly, the experimental set up of this paper can be a case study for carrying out VR-related experiments in post COVID-19 times, where participant hygiene is of maximum importance. The Logitech VR Ink was used in the interest of evaluating stylus-shaped VR controllers as an alternative to gaming controllers.

\section{METHOD}

For determine how product designers experience VR-based ideation, the following experiment was carried out. 10 Product development (industrial design) graduates ( 8 male, 2 female) aged between 23 and 31, three of which were left-handed (Table 1), were asked to draw a respirator mask. They were selected to have very little to zero experience with VR sketching, no experience with VR sketching, but years of experience with traditional CAD design. The respirator mask was chosen as a subject because it is an organically shaped wearable product, which are favorable characteristics for designing in VR (Nunes et al., 2017).

Table 1. Relevant participant demographics

\begin{tabular}{lccc}
\hline & Count & \% & Designs (figure 4) \\
\hline Male & 8 & $\mathbf{8 0}$ & $1,2,4,5,6,8,9,10$ \\
Female & 2 & $\mathbf{2 0}$ & 3,7 \\
Experienced with VR & 4 & $\mathbf{4 0}$ & $1,2,4,10$ \\
Left-handed & 3 & $\mathbf{3 0}$ & $4,5,8$ \\
Opted for paper sketching & 6 & $\mathbf{6 0}$ & $1,4,7,8,9,10$ \\
Visibly nervous & 1 & $\mathbf{1 0}$ & 8 \\
Done before 30 min. & 2 & $\mathbf{2 0}$ & 5,6 \\
\hline
\end{tabular}

Considering the COVID-19 pandemic, the participants were first informed about all the measures taken to keep the experimental environment clean and safe (Figure 1). After a 3-minute pre-test demographic survey (age, experience with design, experience with VR et cetera) they were taught the basics of Gravity Sketch. For this, they were shown eight short tutorial videos (9 minutes in total) of the Gravity Sketch YouTube channel (Gravity Sketch, 2020). These were selected for teaching a certain, basic workflow where you only work with lines and surfaces (Figure 2). After this tutorial the participants were shown two examples of the same workflow from two Gravity Sketch experts on Instagram: Nick P. Baker (Baker, 2018) and Fed Rios (Rios, 2019).
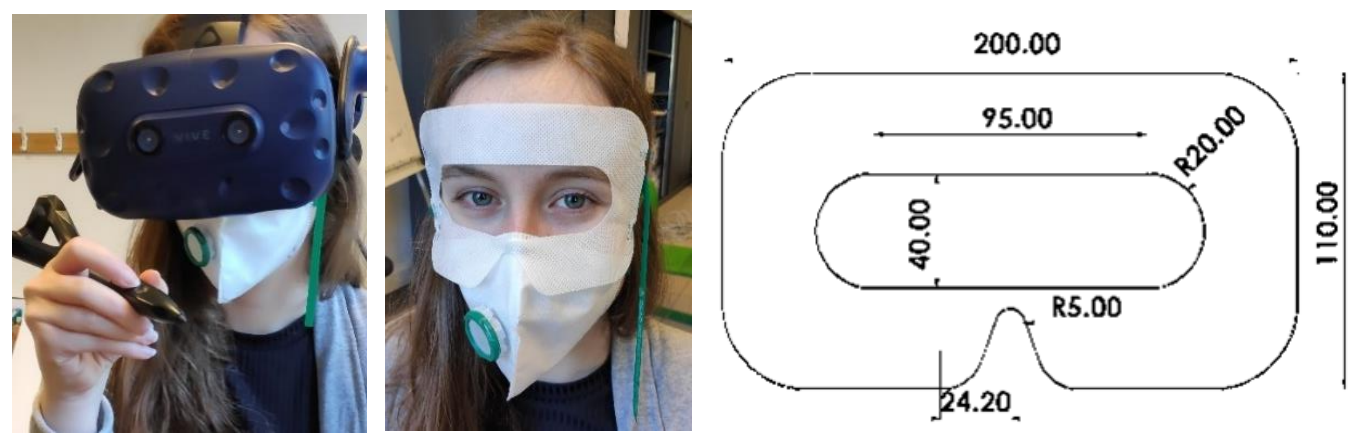

Figure 1. Protecting the participants health during the COVID-19 crisis: Preventive protective equipment: FFP3 protective respirator (left) and lasercutted polypropylene (PP) face barrier (right). Additionally, the equipment was disinfected in between participants.

To make sure they had the ability create something worthwhile within thirty minutes, the decision was made to not show them all the tools available within Gravity Sketch. The workflow that was shown to them (Figure 2) consisted of sketching lines first, drawing surfaces in between these lines and editing them afterwards if needed. This was done so that the participants were able to focus on their own creativity and the respirator mask design instead of focusing on having to learn and choose from a multitude of available software tools. 

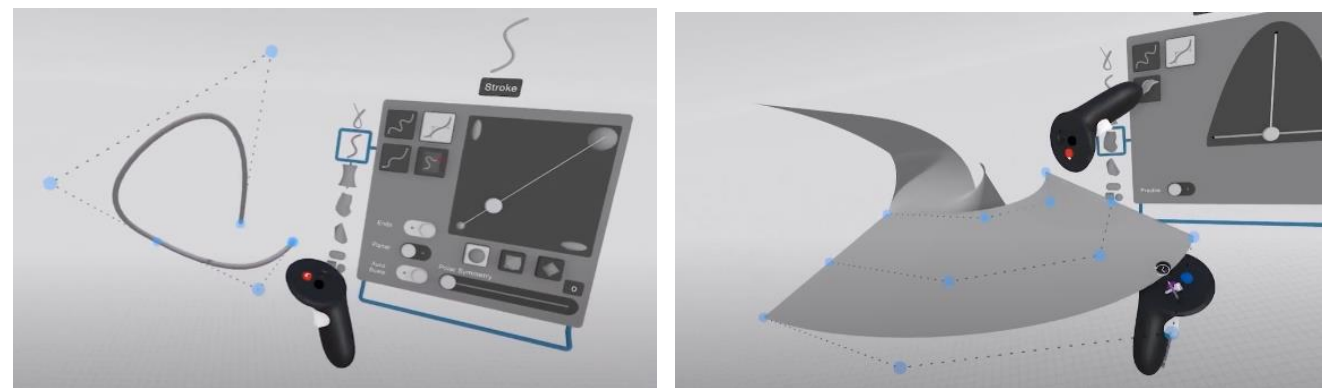

Figure 2. Screenshots from the Gravity Sketch tutorials depicting the simplified workflow: Lines (left) and surfaces (right)

For mimicking their natural workflow, the participants could choose whether they wanted to brainstorm on their design on paper for five minutes beforehand. 6 out of 10 participants opted for this. Either way, they had 5 minutes for brainstorming and 30 minutes for using Gravity Sketch to 3D model their respirator mask design. The design session was partially video recorded and completely screen recorded using the built-in camera function from Gravity Sketch and the screen capture program OBS Studio (Jim, n.d.). An example video was added to the appendix. In Figure 3 a participant in action is shown, the researchers could follow their progress on the screen.
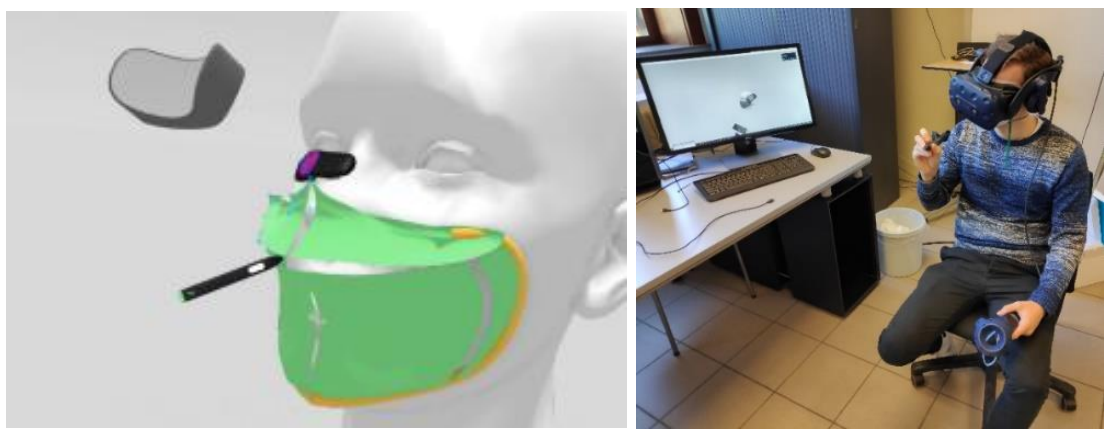

Figure 3. Screenshot from the screen recordings in Gravity Sketch (left), participant 3Dsketching a mask with the HTC Vive pro.

Lastly, the VR Ink stylus (Figure 4) was only briefly explained in advance to the participants since there are no tutorials for it at that time. However, the digital UI of the Logitech VR Ink stylus in Gravity Sketch looks similar to the one of the HTC Vive controller handsets and the participants were allowed to ask for help at any time. Consequently, the first five minutes of every session were spent explaining the basic functions and layout of the VR Ink.

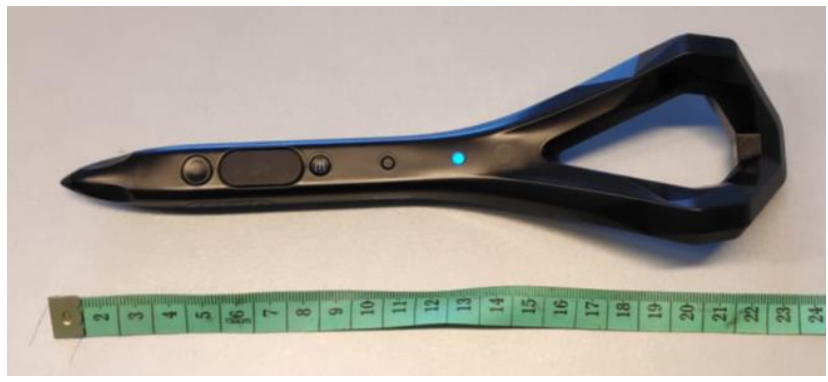

Figure 4. Logitech VR ink Pilot Edition ("Logitech VR Ink Pilot Edition - Ink Differently", n.d.). Contains two force sensitive controls (index finger and stylus tip), clickable $2 D$ touch strip and dual sided grip grab buttons. 232x64x42mm, Weight $68 \mathrm{~g}$

After this session, they were asked to fill out a larger survey containing a few agree - disagree statements, a NASA TLX survey (Hart, 2006) for measuring workload and a PSSUQ questionnaire (Lewis, 2002) for measuring usability. The experiment ends with a verbal debrief (5 min.) for each participant, to give them the chance to furtherly explain their reasoning. The debrief served as a qualitative substantiation of the results and possibly fixing mistakes in reasoning. When the conversation came to a hold, a why question was posed. Additionally, five questions were prepared to guide the conversation: 'How was it?', 'How does this compare to your initial expectations?', 'How was the pen?', 
'Was there anything you felt was missing?' and 'Would you use it in your own workflow?'. The questions were followed by laddering 'why' statements, giving the participants ample time to reflect and talk about their experiences. The responses were recorded and transcribed.

\section{RESULTS}

\subsection{Survey data}

The reactions on the statements (Table 2) infer that the participants saw VR as intuitive, something they want for themselves and fun. There was doubt whether VR based sketching is the future of 3D modelling, but they certainly did not write it off as improbable. This was also reflected in the debrief. The participants liked the standard HTC Vive controller better when compared to the VR Stylus.

Table 2. Perceived value of 6 statements about the user experience with scores from "1, strongly disagree" to " 5 , strongly agree". $(n=10)$

\begin{tabular}{|c|c|c|c|}
\hline \multirow{2}{*}{ Statements } & \multicolumn{3}{|c|}{ Results } \\
\hline & AVG & $\mathrm{SD}$ & Distribution \\
\hline $\begin{array}{l}\text { VR-based 3D modelling is } \\
\text { intuitive }\end{array}$ & 3.9 & 1.20 & -1 \\
\hline $\begin{array}{l}\text { VR-based 3D modelling is } \\
\text { something that I want for }\end{array}$ & 4.0 & 0.67 & $\square \mid$ \\
\hline myself & & & $\begin{array}{lllll}1 & 2 & 3 & 4 & 5\end{array}$ \\
\hline VR-based 3D modelling is fun & 4.5 & 0.71 & $\mid$ \\
\hline $\begin{array}{l}\text { VR-based 3D modelling is the } \\
\text { future of } 3 D \text { modelling }\end{array}$ & 3.6 & 0.52 & \\
\hline A 3D Stylus is comfortable to use & 1.8 & 0.79 & $\mid$ \\
\hline $\begin{array}{l}\text { A standard HTC Vive controller } \\
\text { is comfortable to use }\end{array}$ & 3.5 & 0.97 & $=\left.\left.\right|_{1=34}\right|_{5}$ \\
\hline
\end{tabular}

The NASA TLX (Raw TLX was used) survey (Hart, 2006) resulted in an average score of 49/100 (SEM $=3.4$ ), which is considered neither high nor low in terms of effort. The dominant factors are mental demand and performance pressure (Figure 5). This was reflected during the debriefing, the participants often specified that they were 'just now getting the hang of it'. The PSSUQ survey (Lewis, 2002) on usability reflects these results with an overall mean score of 3.6/7 (SEM =0.3). The participants neither liked nor disliked the usability of the VR-CAD system (Figure 6).
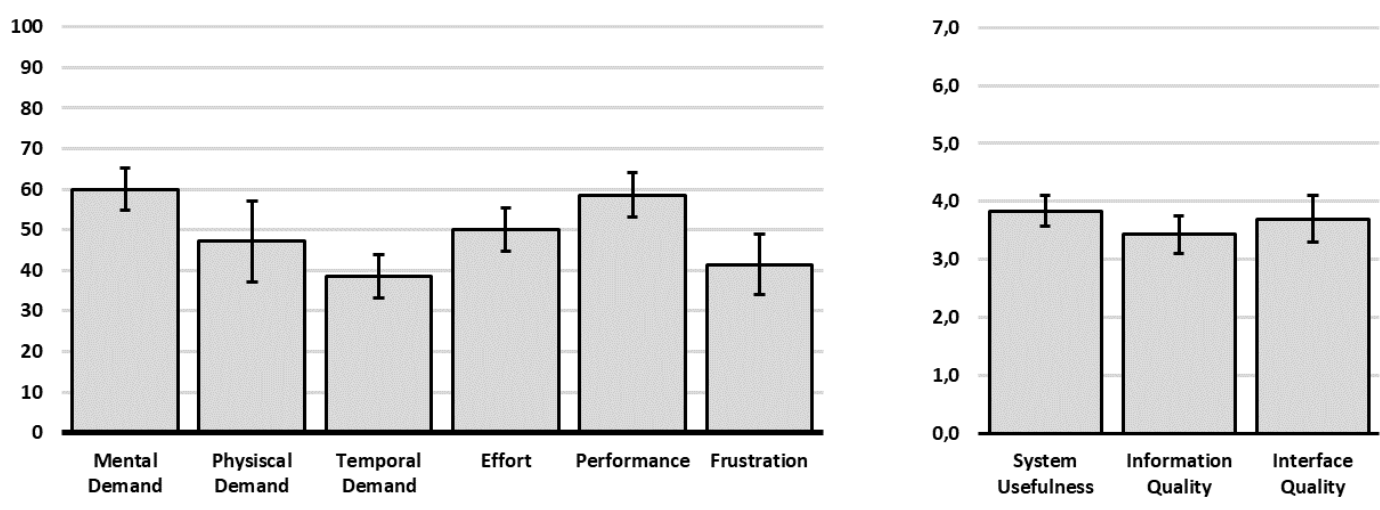

Figure 5. Left: NASA TLX subcategory scores and their standard errors of means (SEM) $(n=10)$ from "0, low demand" to "100, high demand". Right: The VR Stylus PSSUQ subcategory results and their standard errors of means $(S E M)(n=10)$ from ' 0 , bad usability' to '7, good usability'. 


\subsection{User Experience (Debrief)}

The designers were positive about their overall experience. They mentioned that they were surprised about the intuitiveness of VR-based 3D modeling and they liked the quality of the respirator mask that they were able to design after only half an hour (Figure 6). Basically, the difficulty for them was using the VR controllers, the Gravity Sketch UI and, for some, using an HMD for the very first time. However, after having to browse the menu to find the right tool, most of them mentioned that it was fun to be able to draw in a digital, immersive space. The act of VR sketching itself required very little mental effort for them. Three participants did mention being anxious at first about the quality of their result. This was because they were shown examples of VR modeling professionals, which were naturally a lot more complete. They thought they had to compete with that, not expecting that there is a certain learning curve to be familiar with the system.

When asked what stage within their own workflow VR-CAD could serve as a replacement, common answers were: ideation sketching, clay modeling, foam modeling and presentation sketching. None of the participants indicated that it could replace screen-based CAD modeling. One of the participants specified that these parts of the design method are parts where you must be able to design very fast, creating models that primarily serve as visual representations. "The fact alone that there is now an 'undo'-button for the ideation phase is exciting", one of the participants said. 90\% of the participants verbally reacted positively to wanting to use this technology in their own workflow.
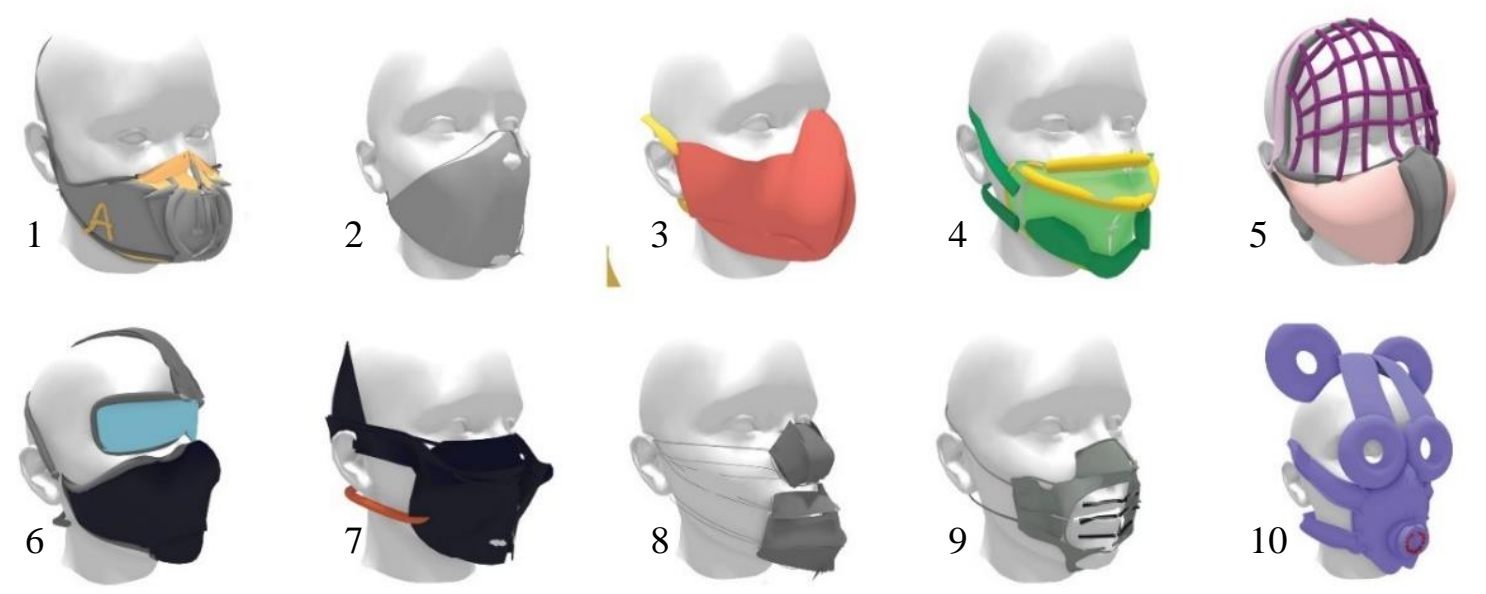

Figure 6. The design results of ten product designers, trying out Gravity Sketch VR for the first time using the Logitech VR Ink (Pilot Edition) stylus and HTC Vive Pro (30 minutes).

During the debrief, everyone had strong opinions about the VR stylus. The participants like the idea of using a pen in VR but they felt disappointed with the product. This paper is more about user overall experience and less about the usability and ergonomics of the pen. However, the comments about the stylus are essential to understanding the survey data.

The participants had high expectations about the pen. Being trained designers themselves, they expected that the pen would be intuitive to them, but generally it was not. The first commonly occurring comment the participants mentioned was the number of small buttons placed all over the pen. While there is an understandable need for replacing a standard VR controllers' button interface, it was difficult for the participants to reach all the necessary functions. Two participants mentioned the fact that it is rather unintuitive to have to control objects with buttons on a pen. They proposed a system where the pen would be used in combination with something like a keyboard, where all the buttons and menus are on the non-drawing hand.

The second comment was the weight being at the back of the pen: half of the participants spoke about a strong imbalance in the hand because of this. In relation to this, although the VR Ink intentionally looks like a traditional pen, $90 \%$ of the participants had to be corrected in the way they initially held the pen (Figure 7). The right way of holding it can be seen in Figure 1. A final comment that almost everybody mentioned about the pen was about the size: Some participants thought the pen was too big to hold, others thought the pen was too small. More than half of the subjects mentioned that they would rather use two traditional VR controllers. 


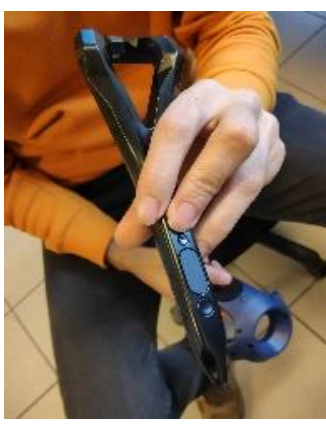

a

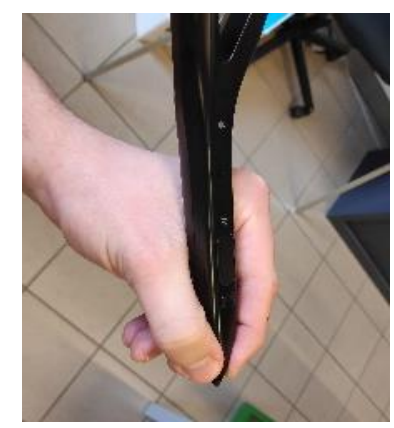

b

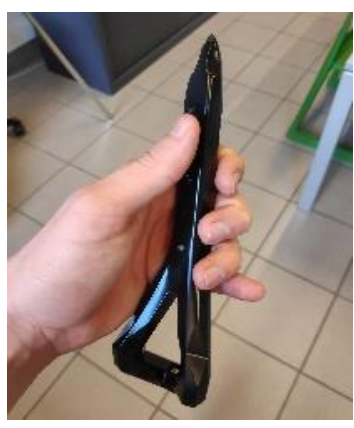

$\mathrm{c}$

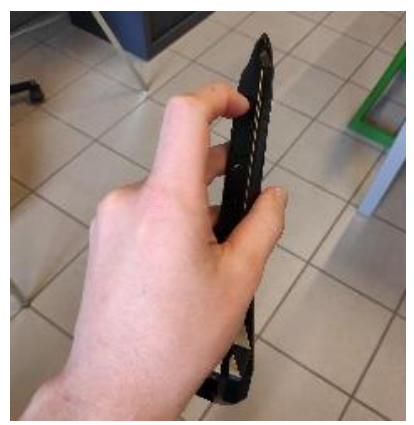

d

Figure 7. Different incorrect ways in which the stylus was initially held by one or more participants. a: Using their middle finger operate the buttons on top; $b$ : Holding it too close to the tip of the pen; $c$ : Holding it in the palm of your hand, much like the traditional controller and $d$ : Holding it like a TV remote.

Insights like these can be used by industrial designers for improving the physical interface for future virtual 'in-air' drawing XR controllers.

\section{DISCUSSION}

The stylus should not be considered written off based on this paper alone. Arguably, the main advantage of the VR stylus was not utilized during this experiment, namely the ability to draw on physical surfaces. This stylus was an early prototype and the tip of the pen arguably was not optimally developed yet, so the participants were instructed not to do so. Additionally mid-air sketching induces the Heisenberg effect (Wolf et al., 2020), where pushing a button inherently causes a certain inaccuracy. We believe a better use case for this VR pen will arrive within the next year, namely the use of this technology in combination with the next generation of augmented reality (AR) systems. Until then, a usability study focusing on VR styli is less relevant. That said, these ten designers generally reacted negatively towards it, which has likely influenced the NASA TLX and PSSUQ results. Participants indicated that they would have likely answered the post-test surveys more positively if the experiment would have been performed without the stylus.

Because of the low participant count and the stylus related frustrations, it is difficult to make concrete statements about VRAD as a whole. Participants indicated that their experience would have been more positive if the stylus was not in the picture, but it is impossible to know just how much this influenced the results. However, using the survey data as a benchmark, the qualitative debrief can serve as a source of insights to further future research on the topic. All participants seemed to enjoy partaking in this experiment and were intrigued with the possibilities of VRAD. Most were talkative and had big ideas about the future of design. The atmosphere was laid back because the task was open to their own interpretation and easy to complete within the time given. The former was reflected in some of the 'funnier' designs displayed in Figure 4. Unlike the NASA TLX and the compounded agree-disagree survey, the use of a PSSUQ questionnaire did not give real insights in this study. This might have been preventable by using a TAM questionnaire instead, like Özgen et al. did (Özgen et al., 2019).

Participants liked the idea of using VRAD in their own workflow for ideation. Based on the resulting concepts generated during the first 30 minutes conclusions can be made about the efficiency and intuitiveness of the tool. Whether or not it could be used for other workflow steps (e.g. anything but ideation) cannot be deduced since only a few tools were presented to the participants to keep it simple. In terms of future research about later stage VRAD design, authors recommend adding a few tools to the list to allow more precision.

Lastly, performing a VR-related experiment post COVID-19 lockdown comes with challenges. A number of measures were taken to make sure the test subjects were willing to participate, and these measures were definitely appreciated. There are also a few advantages to using maximal face covering protective equipment. For example, one of the participants did have some face-sweat, but it did not come into contact with the foam on the HMD. Even if it would have, the next participant would not notice because the polypropylene fabric blocks any moisture. Consequentially any liquid or soap residue (or scent) went unnoticed because the skin (and the nose) were completely covered. A certain disadvantage 
is the added tension from the face masks in combination with wearing a VR headset. All participants felt relieved when they could finally take everything off.

\section{CONCLUSION \& FUTURE WORK}

The fact that these designers were able to make a 3D product sketch that looks like an actual product, in 30 minutes, with no prior knowledge of the software is remarkable. The conclusion of this paper is twofold. Firslty, although the participants were not convinced on the VR stylus, the participants indicated that VR-based 3D modeling is fun and something that they would like to use in their own workflow. Ideation, concept design and visual presentation are brought forward as workflow tasks wherein VR sketching would be most effective. Secondly, participants experienced VRAD as a quick and intuitive tool for 3D ideation. They personally felt performant and they enjoyed the process. As of now, VRAD is not seen as an alternative to neither sketching nor CAD. Instead, new users experience it being a new tool that can be positioned either parallel to or in between ideation sketching, clay modeling and detailed CAD design.

This paper validates and builds on the work of Joundi et al. (Joundi et al., 2020), on all but one point; they state in their conclusion that they did not use VR as a form finding tool because it allegedly has a steep learning curve. This paper disproves that by explicitly using it as a form finding tool in a similar setting. While it is true that there is a certain learning curve for high end, detailed visualization, participants were generally surprised about what could be accomplished within their first 40 minutes. In terms of validity for shape ideation, the paper validates the findings Oti and Crilly (Oti and Crilly, 2020) on early stage designing with VR. In terms of personal effectiveness and enjoyment these findings confirm those of Özgen et al and Maurya et al (Maurya et al., 2020; Özgen et al., 2019).

It would be interesting to recreate this experiment, including a comparison between different kinds of controllers. Secondly, it is worth studying ergonomic and usability requirements of next generation controllers to be used for industrial design purposes. Additionally, using 'enjoyment' as a parameter for this kind of study seems obvious but was considerably telling. Lastly, for studying user experience, the workflow of the participants was predetermined (only curves and surfaces) to make sure that they were able to create something worthwhile within 30 minutes. This means that this experiment does not present a definite claim about the 'natural' workflow of each designer. A follow-up study could be one that focuses on the 'natural' workflow of designers who are already experienced in using VR-based CAD software. Although further research is necessary, it is likely that adoption of VRAD in an industrial design workflow would be beneficial.

\section{REFERENCES}

Adenauer, J., Israel, J.H. and Stark, R. (2013), “Virtual Reality Technologies for Creative Design”, CIRP Design 2012, Springer London, London, pp. 125-135.

Bae, S.H., Balakrishnan, R. and Singh, K. (2008), "Ilovesketch: As-natural-as-possible sketching system for creating 3D curve models", UIST 2008 - Proceedings of the 21st Annual ACM Symposium on User Interface Software and Technology, pp. 151-160.

Baker, N.P. (2018), “nickpbaker”, available at: https://www.instagram.com/p/BgHycOlDeQ7/ (accessed 17 May 2020).

Bakker, T., Verlinden, J., Abbink, D. and Deventer, R. van. (2017), "Development of a Haptic Device with Tactile and Proprioceptive Feedback for Spatial Design Tasks”, 2017 IEEE International Symposium on Mixed and Augmented Reality (ISMAR-Adjunct), IEEE, pp. 223-228.

Balzerkiewitz, H.-P. and Stechert, C. (2020), "the Evolution of Virtual Reality Towards the Usage in Early Design Phases", Proceedings of the Design Society: DESIGN Conference, Vol. 1, pp. 91-100.

Berg, L.P. and Vance, J.M. (2017), "Industry use of virtual reality in product design and manufacturing: a survey", Virtual Reality, Springer London, Vol. 21 No. 1, available at:https://doi.org/10.1007/s10055-016-0293-9.

Bordegoni, M., Ferrise, F., Covarrubias, M. and Antolini, M. (2010), "Haptic and sound interface for shape rendering", Presence: Teleoperators and Virtual Environments, Vol. 19 No. 4, pp. 341-363.

Bourdot, P., Convard, T., Picon, F., Ammi, M., Touraine, D. and Vézien, J.M. (2010), "VR-CAD integration: Multimodal immersive interaction and advanced haptic paradigms for implicit edition of CAD models", CAD Computer Aided Design, Vol. 42 No. 5, pp. 445-461.

Cass, S. (2019), “Tilt Brush: The Killer App for VR”, available at: https://spectrum.ieee.org/consumerelectronics/audiovideo/tiltbrush-the-killer-app-for-vr (accessed 17 May 2020). 
Chu, C.C.P., Dani, T.H. and Gadh, R. (1997), "Multi-sensory user interface for a virtual-reality-based computeraided design system", CAD Computer Aided Design, available at: https://doi.org/10.1016/S00104485(97)00021-3.

Couche, G., Paslier, P., Sosanya, O. and Fuentes, D.P. (2014), “|Gravity Sketch”, available at: https://www.rca.ac.uk/research-innovation/innovationrca/innovationrca-start-ups/gravity/ (accessed 17 May 2020).

Drey, T., Gugenheimer, J., Karlbauer, J., Milo, M. and Rukzio, E. (2020), "VRSketchIn : Exploring the Design Space of Pen and Tablet Interaction for 3D Sketching in Virtual Reality", pp. 1-14.

Fiorentino, M., De Amicis, R., Monno, G. and Stork, A. (2002), "Spacedesign: A mixed reality workspace for aesthetic industrial design", Proceedings - International Symposium on Mixed and Augmented Reality, ISMAR 2002, No. February, pp. 86-96.

Fuge, M., Yumer, M.E., Orbay, G. and Kara, L.B. (2012), "Conceptual design and modification of freeform surfaces using dual shape representations in augmented reality environments", CAD Computer Aided Design, Elsevier Ltd, Vol. 44 No. 10, pp. 1020-1032.

Van Goethem, S., Watts, R., Dethoor, A., Van Boxem, R., van Zegveld, K., Verlinden, J. and Verwulgen, S. (2020), “The Use of Immersive Technologies for Concept Design”, Advances in Intelligent Systems and Computing, Vol. 1217 AISC, pp. 698-704.

Gravity Sketch. (2020), "VR Tutorials", available at: https://www.youtube.com/playlist?list=PLdcetkoP2x8RV_EGwBhQaAom9dqq4Bii0 (accessed 18 May 2020).

Häggman, A., Tsai, G., Elsen, C., Honda, T. and Yang, M.C. (2015), “Connections Between the Design Tool, Design Attributes, and User Preferences in Early Stage Design”, Journal of Mechanical Design, Vol. 137 No. 7, available at: https://doi.org/10.1115/1.4030181.

Hart, S.G. (2006), "NASA-task load index (NASA-TLX); 20 years later", Proceedings of the Human Factors and Ergonomics Society, pp. 904-908.

Hummels, C. and Stappers, P.J. (1998), "Meaningful gestures for human computer interaction: beyond hand postures", Proceedings Third IEEE International Conference on Automatic Face and Gesture Recognition, IEEE Comput. Soc, pp. 591-596.

Ingrassia, T. and Cappello, F. (2009), “VirDe: A new virtual reality design approach”, International Journal on Interactive Design and Manufacturing, Vol. 3 No. 1, pp. 1-11.

Israel, J.H., Wiese, E., Mateescu, M., Zöllner, C. and Stark, R. (2009), “Investigating three-dimensional sketching for early conceptual design-Results from expert discussions and user studies", Computers and Graphics (Pergamon), Vol. 33 No. 4, pp. 462-473.

Jackson, B. and Keefe, D.F. (2016), "Lift-Off: Using Reference Imagery and Freehand Sketching to Create 3D Models in VR", IEEE Transactions on Visualization and Computer Graphics, IEEE, Vol. 22 No. 4, pp. 1442-1451.

Jim. (n.d.). "OBS - Open Broadcaster Software”, available at: https://obsproject.com/.

Joundi, J., Christiaens, Y., Saldien, J., Conradie, P. and De Marez, L. (2020), “an Explorative Study Towards Using Vr Sketching As a Tool for Ideation and Prototyping in Product Design”, Proceedings of the Design Society: DESIGN Conference, Vol. 1, pp. 225-234.

Krichenbauer, M., Yamamoto, G., Taketom, T., Sandor, C. and Kato, H. (2018), “Augmented Reality versus Virtual Reality for 3D Object Manipulation”, IEEE Transactions on Visualization and Computer Graphics, Vol. 24 No. 2, pp. 1038-1048.

Lawson, G., Salanitri, D. and Waterfield, B. (2016), "Future directions for the development of virtual reality within an automotive manufacturer", Applied Ergonomics, Elsevier, Vol. 53, pp. 323-330.

Lewis, J.R. (2002), "Psychometric evaluation of the PSSUQ using data from five years of usability studies", International Journal of Human-Computer Interaction, Vol. 14 No. 3-4, pp. 463-488.

"Logitech VR Ink Pilot Edition - Ink Differently". (n.d.). , available at: https://www.logitech.com/enroeu/promo/vr-ink.html (accessed 17 May 2020).

Maurya, S., Arai, K., Moriya, K., Arrighi, P.A. and Mougenot, C. (2019), “A mixed reality tool for end-users participation in early creative design tasks", International Journal on Interactive Design and Manufacturing, Springer Paris, Vol. 13 No. 1, pp. 163-182.

Maurya, S., Mougenot, C. and Takeda, Y. (2020), "Impact of mixed reality implementation on early-stage interactive product design process", Journal of Engineering Design, available at: https://doi.org/10.1080/09544828.2020.1851662.

Milosevic, B., Bertini, F., Farella, E. and Morigi, S. (2016), “A SmartPen for 3D interaction and sketch-based surface modeling”, International Journal of Advanced Manufacturing Technology, The International Journal of Advanced Manufacturing Technology, Vol. 84 No. 5-8, pp. 1625-1645.

Nee, A.Y.C., Ong, S.K., Chryssolouris, G. and Mourtzis, D. (2012), “Augmented reality applications in design and manufacturing”, CIRP Annals - Manufacturing Technology, CIRP, Vol. 61 No. 2, pp. 657-679.

Nunes, M.L., Pereira, A.C. and Alves, A.C. (2017), "Smart products development approaches for Industry 4.0”, Procedia Manufacturing, Elsevier, Vol. 13, pp. 1215-1222. 
Oti, A. and Crilly, N. (2020), "Immersive 3D sketching tools: Implications for visual thinking and communication", Computers \& Graphics, Elsevier Ltd, No. xxxx, available at: https://doi.org/10.1016/j.cag.2020.10.007.

Özgen, D.S., Afacan, Y. and Sürer, E. (2019), "Usability of virtual reality for basic design education: a comparative study with paper-based design”, International Journal of Technology and Design Education, Springer Netherlands, No. 0123456789, available at: https://doi.org/10.1007/s10798-019-09554-0.

Piumsomboon, T., Clark, A., Billinghurst, M. and Cockburn, A. (2013), "User-Defined Gestures for Augmented Reality BT - Human-Computer Interaction - INTERACT 2013”, Human-Computer Interaction INTERACT 2013, Vol. 8118 No. Chapter 18, pp. 282-299.

"Quill VR illustration and animation tool built to empower artists and creators". (n.d.)., available at: https://quill.fb.com/ (accessed 17 May 2020).

Reipschläger, P. and Dachselt, R. (2019), "Designar: Immersive 3D-modeling combining augmented reality with interactive displays", ISS 2019 - Proceedings of the 2019 ACM International Conference on Interactive Surfaces and Spaces, pp. 29-41.

Rieuf, V., Bouchard, C., Meyrueis, V. and Omhover, J.-F. (2017), "Emotional activity in early immersive design: Sketches and moodboards in virtual reality", Design Studies, Vol. 48, pp. 43-75.

Rios, F. (2019), "fedriosdesign - hiking backpack", available at: https://www.instagram.com/p/B1A6HOWHvo7/ (accessed 17 May 2020).

Sachs, E., Roberts, A. and Stoops, D. (1991), “3-Draw: a tool for designing 3D shapes”, IEEE Computer Graphics and Applications, Vol. 11 No. 6, pp. 18-26.

Schkolne, S., Pruett, M. and Schröder, P. (2001), "Surface drawing”, Proceedings of the SIGCHI Conference on Human Factors in Computing Systems - CHI '01, ACM Press, New York, New York, USA, pp. 261-268.

Thorsteinsson, G. and Page, T. (2007), "Creativity in Technology Education Facilitated through Virtual Reality Learning Environments: A Case Study.”, Journal of Educational Technology, Vol. 3 No. 4, pp. 74-87.

Tovey, M. (1997), "Styling and design: Intuition and analysis in industrial design”, Design Studies, Vol. 18 No. 1 , pp. 5-31.

Wang, S., He, W., Zheng, B., Feng, S., Wang, S., Bai, X. and Billinghurst, M. (2019), "Holding virtual objects using a tablet for tangible 3D sketching in VR", Adjunct Proceedings of the 2019 IEEE International Symposium on Mixed and Augmented Reality, ISMAR-Adjunct 2019, Vol. 6, pp. 156-157.

Wolf, D., Gugenheimer, J., Combosch, M. and Rukzio, E. (2020), "Understanding the Heisenberg Effect of Spatial Interaction: A Selection Induced Error for Spatially Tracked Input Devices”, Chi 2020, Vol. 20, pp. 1-10. 